\title{
The Severe Asthma Questionnaire: sensitivity to change and minimal clinically important difference
}

\author{
To the Editor:
}

The Severe Asthma Questionnaire (SAQ) is a validated measure of the health-related quality of life of people living with severe asthma [1]. The minimal clinically important difference (MCID) of the SAQ has not been calculated. The MCID is useful for representing clinical as opposed to statistical significance. There are two main ways of calculating the MCID. Distribution methods define the MCID in terms of the relationship between the distribution of scores and mean change score. These methods are purely statistical and the relationship and formulae that constitute the MCID is determined by convention. By contrast, the anchor method [2] defines the MCID in terms of an independent anchor or criterion. When the anchor is the patient's perception of a just noticeable difference in their condition, then the anchor method has two advantages over the distribution method. First, the MCID is defined by a criterion and, therefore, has criterion validity rather than being only a convention. Second, the MCID is defined in terms of the patient's perception of treatment, and the patient's perception of their treatment is recognised as being an important outcome for clinical decision-making [2]. In this letter we present the MCID of the SAQ using the anchor method.

110 patients were recruited from six UK specialist asthma centres who were initiating a National Institute of Health and Care Excellence approved biologic treatment as part of usual care (62\% female, mean age 49 years, mean forced expiratory volume in $1 \mathrm{~s} 67 \%$ of predicted). Participants completed the SAQ at baseline and completed the SAQ and a Global Rating of Change (GRoC) questionnaire [3] at 4, 8, 12 and 16 weeks after starting treatment. Patients responded to the 16 items of the SAQ using a 7-point scale, and responses were scored to produce an overall SAQ score from the mean of 16 items, and three subscale scores (My Life, My Mind, My Body) from the means of subsets of those 16 items [4]. In addition, patients provided a single response to a 100-point Borg-type rating of global quality of life, the SAQ-global. Questionnaires with $>10 \%$ missing items were deemed invalid. Baseline mean \pm SD scores demonstrated significant health-related quality of life impairment across all aspects of the SAQ: $3.65 \pm 1.49$ for the SAQ, 3.74 \pm 1.62 for My Life subscale, 3.78 \pm 1.78 for My Mind subscale, 3.34 \pm 1.51 for My Body subscale and $45.50 \pm 23.67$ for the SAQ-global. Questionnaire change scores were calculated by subtracting the baseline score from the follow-up score, so that positive change scores indicated improvement in quality of life. There was a significant $(\mathrm{p}<0.01)$ improvement between baseline and 4 weeks for the SAQ, the subscales of the SAQ and the SAQ-global, and improvement was maintained for subsequent weeks.

Patients were asked to rate the GRoC in terms of improvement by circling a statement "which best describes how you feel since starting your new treatment for your asthma". We analysed GRoC responses into any one of six categories: 1) "any degree of deterioration", 2) "no change", 3) "a little better", 4) "somewhat better" or "moderately better", 5) "a good deal better" and 6) "a great deal better". Perceived change of "a little better" was defined as the point of the MCID following normal practice. In order to take full advantage of the repeated measures design, we used all available data where a GRoC rating was accompanied by a valid questionnaire change score over the 16-week study but excluded scores where the GRoC category was the repeat or deterioration of the previous week, as such scores could be less reliable due to increased recall bias caused by the intervening experiences. The study received ethical approvals

@ERSpublications

The Severe Asthma Questionnaire (SAQ) is sensitive to change and clinically significant improvement was detected within 4 weeks of starting biologic therapy. The MCID of the SAQ is 0.5 and of the SAQ-global is 11. https://bit.ly/3poJqcG

Cite this article as: Masoli M, Lanario JW, Hyland ME, et al. The Severe Asthma Questionnaire: sensitivity to change and minimal clinically important difference. Eur Respir J 2021; 57: 2100300 [https:// doi.org/10.1183/13993003.00300-2021]. 
TABLE 1 Change scores for those reporting different degrees of global rating of change

\begin{tabular}{lccccc} 
Description of change (GRoC scale $\mathbf{0}-\mathbf{5})$ & SAQ & SAQ My life & SAQ My mind & SAQ My body & SAQ-global \\
\hline Any degree of deterioration (-5 to $-\mathbf{1})$ & $-0.41 \pm 1.15(4)$ & $0.06 \pm 0.88(4)$ & $-1.23 \pm 1.45(4)$ & $-0.50 \pm 1.50(4)$ & $-5.00 \pm 15.81(4)$ \\
No change (0) & $-0.32 \pm 0.97(34)$ & $-0.30 \pm 1.04(35)$ & $-0.42 \pm 1.21(35)$ & $-0.25 \pm 1.32(35)$ & $0.95 \pm 16.78(37)$ \\
“A little better" (1) (MCID) & $0.54 \pm 0.94(28)$ & $0.67 \pm 0.90(28)$ & $0.43 \pm 1.11(28)$ & $0.45 \pm 1.38(28)$ & $10.89 \pm 14.91(28)$ \\
“Somewhat better" and “moderately better" (2 or 3) & $0.92 \pm 1.04(34)$ & $0.90 \pm 1.01(33)$ & $0.90 \pm 1.00(33)$ & $0.84 \pm 1.82(33)$ & $17.41 \pm 16.96(34)$ \\
“A good deal better" (4) & $1.23 \pm 1.10(33)$ & $1.11 \pm 1.27(33)$ & $1.20 \pm 1.22(33)$ & $0.99 \pm 1.27(33)$ & $19.18 \pm 20.38(33)$ \\
“A great deal better" (5) & $2.19 \pm 1.05(27)$ & $2.19 \pm 1.19(26)$ & $2.32 \pm 1.52(26)$ & $2.15 \pm 1.70(26)$ & $31.83 \pm 19.00(29)$ \\
\hline
\end{tabular}

Data are presented as mean \pm SD (n). GRoC: Global Rating of Change; SAQ: Severe Asthma Questionnaire; MCID: minimal clinically important difference.

from the Research Ethics Committee/Health Research Authority (REC reference: 19/WA/0011, IRAS project ID: 250167) and was sponsored by University Hospitals Plymouth NHS Trust.

The coronavirus disease 2019 pandemic reduced data collection at later time points. Of the 110 patients recruited, 107 provided valid change scores at week 4,74 at week 8,30 at week 12 and 49 at week 16 . Of the 28 judgements that were used to assess the MCID of the SAQ, 18 and 10 came from weeks 4 and 8 , respectively. No additional judgements for MCID calculations were provided at weeks 12 or 16 . The 110 patients recruited provided a total of 799 judgements that are presented in table 1.

These data show that the MCID for the SAQ is 0.5 and for the SAQ-global is 11. The MCID for the subscales of the SAQ are similar to that of the SAQ. Based on data reported elsewhere [1], the standard error of measurement [5] gives an MCID of 0.5 for the SAQ and 6.0 for the SAQ-global. The standard error of measurement method provides an estimate of statistical discrimination and should not normally be greater than that provided by the anchor method. An MCID of 0.5 is reported by the authors of another asthma questionnaire, the Asthma Quality of Life Questionnaire (AQLQ) [6] that, like the SAQ, asks patients to rate on a 7-point scale. However, the similarity in results should be interpreted cautiously as there are some differences in the anchor methodology, and the assessment of the MCID of the AQLQ was based on the mean of only 10 judgements. Other asthma questionnaires, such as the St George's Respiratory Questionnaire [7] have other forms of response format and are therefore not comparable.

In addition to providing the MCID for the SAQ, our data provide information about the multiples of the MCID that are equivalent to larger degrees of perceived change. A "great deal better" is approximately four times the MCID for the SAQ and three times the MCID for the SAQ-global. A "good deal better" is approximately twice the MCID for the SAQ and twice the MCID for the SAQ-global. These additional values can be used to assess the clinical significance of changes that are much greater than the MCID, such as in super-responders.

The SAQ was developed with patients who contributed as partners to the content, wording and format of the questionnaire $[8,9]$. The 16 items are validated to be used as a single scale (SAQ) [1] or as three subscales (My Life, My Mind, My Body) [4], and the questionnaire also provides a global estimate of quality of life (SAQ-global). In this paper we have presented the MCID for all scoring methods.

Matthew Masoli $\odot^{1}$, Joseph W. Lanario ${ }^{2}$, Michael E. Hyland ${ }^{2,3}$, Andrew Menzies-Gow ${ }^{4}$, Adel H. Mansur $\oplus^{5}$, David Allen ${ }^{6}$, James W. Dodd $\oplus^{7}$, Gemma Hayes ${ }^{8}$, Jose M. Valderas ${ }^{9}$, Sarah E. Lamb ${ }^{9}$ and Rupert C. Jones ${ }^{2}$

${ }^{1}$ Dept of Respiratory Medicine, Royal Devon and Exeter Hospital, University of Exeter, Exeter, UK. ${ }^{2}$ Faculty of Health, University of Plymouth, Plymouth, UK. ${ }^{3}$ Plymouth Marjon University, Plymouth, UK. ${ }^{4}$ Dept of Respiratory Medicine, Royal Brompton Hospital, London, UK. ${ }^{5}$ Dept of Respiratory Medicine, Heartlands Hospital, University Hospitals Birmingham and University of Birmingham, Birmingham, UK. ${ }^{6}$ School of Biological Sciences, Faculty of Biology, Medicine and Health, University of Manchester, Manchester Academic Health Science Centre and NIHR Manchester Biomedical Research Centre, Manchester University Hospitals NHS Foundation Trust, Manchester, UK. ${ }^{7}$ Academic Respiratory Unit, University of Bristol, Southmead Hospital, North Bristol Hospital Trust, Bristol, UK. ${ }^{8}$ Dept of Respiratory Medicine, University Hospitals Plymouth NHS Trust, Plymouth, UK. ${ }^{9}$ College of Medicine and Health, University of Exeter, Exeter, UK.

Correspondence: Matthew Masoli, Dept of Respiratory Medicine, Royal Devon and Exeter Hospital, Barrack Road, Exeter, EX2 5DW, UK. E-mail: matthew.masoli@nhs.net

Received: 17 Dec 2020 | Accepted: 8 Feb 2021

Conflict of interest: M. Masoli reports grants from GlaxoSmithKline (GSK supported this study with an investigator led non-promotional grant, they had no involvement in the study, analysis or write up), personal fees from AstraZeneca (independent data monitoring committee member), personal fees from Novartis (participation in advisory board), 
during the conduct of the study. J.W. Lanario reports grants from GSK, during the conduct of the study; personal fees from Novartis, outside the submitted work. M.E. Hyland reports grants from GSK, during the conduct of the study; personal fees from GSK, outside the submitted work. A. Menzies-Gow reports grants and personal fees from AstraZeneca, personal fees and non-financial support from Teva, and personal fees from Novartis, GlaxoSmithKline, Sanofi, Roche and Vectura, outside the submitted work. A.H. Mansur reports grants, personal fees, non-financial support and other (for various activities that include talks, advisory board meeting, sponsorship for conferences) from NAPP, Cheisi, AstraZeneca, GSK, Sanofi, Teva, outside the submitted work. D. Allen reports personal fees (advisory boards) from AstraZeneca and GSK, personal fees and non-financial support from Teva (speaker fees, and sponsorship support to attend conferences), outside the submitted work. J.W. Dodd has nothing to disclose. G. Hayes has nothing to disclose. J.M. Valderas has nothing to disclose. S.E Lamb was on the Health Technology Assessment (HTA) Additional Capacity Funding Board, HTA End of Life Care and Add-on Studies Board, HTA Prioritisation Group Board and the HTA Trauma Board. R.C. Jones reports grants from GlaxoSmithKline, during the conduct of the study; grants, personal fees and non-financial support from AstraZeneca and GlaxoSmithKline, personal fees and non-financial support from Boehringer Ingelheim, Novartis, Nutricia and OPRI, outside the submitted work.

Support statement: This work was supported by GlaxoSmithKline Investigator sponsored studies (ISS) grant: ISS Number 8994. Funding information for this article has been deposited with the Crossref Funder Registry.

\section{References}

1 Hyland ME, Jones RC, Lanario JW, et al. The construction and validation of the Severe Asthma Questionnaire. Eur Respir J 2018; 52: 1800618.

2 Revicki D, Hays RD, Cella D, et al. Recommended methods for determining responsiveness and minimally important differences for patient-reported outcomes. J Clin Epidemiol 2008; 61: 102-109.

3 Kamper SJ, Maher CG, Mackay G. Global rating of change scales: a review of strengths and weaknesses and considerations for design. J Man Manip Ther 2009; 17: 163-170.

4 Lanario JW, Hyland ME, Menzies-Gow A, et al. Validation of subscales of the Severe Asthma Questionnaire (SAQ) using exploratory factor analysis (EFA). Health Qual Life Outcomes 2020; 18: 336.

5 Wyrwich KW, Wolinsky FD. Identifying meaningful intra-individual change standards for health-related quality of life measures. J Eval Clin Pract 2000; 6: 39-49.

6 Juniper EF, Guyatt GH, Willan A, et al. Determining a minimal important change in a disease-specific quality of life questionnaire. J Clin Epidemiol 1994; 47: 81-87.

7 Jones PW, Quirk FH, Baveystock CM. The St George's respiratory questionnaire. Respir Med 1991; 85 Suppl B: 25-31.

8 Hyland ME, Lanario JW, Pooler J, et al. How patient participation was used to develop a questionnaire that is fit for purpose for assessing quality of life in severe asthma. Health Qual Life Outcomes 2018; 16: 24.

9 Hyland ME, Whalley B, Jones RC, et al. A qualitative study of the impact of severe asthma and its treatment showing that treatment burden is neglected in existing asthma assessment scales. Qual Life Res 2015; 24: 631-639.

Copyright (C)The authors 2021. 